\title{
Preface to special issue on leguminous pulses
}

\author{
Teodardo Calles ${ }^{1}$
}

Published online: 8 December 2016

(c) Springer Science+Business Media Dordrecht 2016

Much still needs to be done to end world hunger and provide food security and nutrition for a swelling global population, expected to reach over 9 billion by 2050 . Humans have consumed pulses for millennia; yet their nutritional, environmental and other benefits are often under-appreciated. To help revert that, the United Nations General Assembly declared 2016 as the International Year of Pulses (IYP). Throughout the IYP, the Food and Agriculture Organization of the United Nations (FAO) has made an effort to bring together a wide spectrum of different actors from diverse sectors to enhance awareness about pulse production and consumption around the world. FAO aimed at creating synergies amongst stakeholders and expanding the role of pulses in sustainable food production.

Pulses is a subgroup of agricultural crops belonging to the Leguminosae family. According to FAO, pulses are legume plants producing edible seeds that are harvested for dry grains and used for human and animal consumption. FAO classification excludes those legume species mainly used for oil extraction [e.g., soybean; Glycine max (L.) Merr.], sowing purposes (e.g., alfalfa; Medicago sativa L.) and when they are used as vegetables (e.g., green peas; Pisum sativum L.).

Pulses, and legumes in general, can play an important role in addressing issues related to food security, environment and health. Pulses are able to biologically fix atmospheric nitrogen and some of them utilize soil-bound phosphorous (e.g., Lupinus albus L.), thus becoming the

Teodardo Calles

teodardo.calles@fao.org

Plant Production and Protection Division (AGP), Food and Agriculture Organization of the United Nations, Viale delle Terme di Caracalla, 00153 Rome, Italy cornerstone of sustainable agriculture. When pulses are included in multiple cropping systems (e.g., crop rotations, intercropping, agroforestry), they provide different services such as improving nutrient cycling and increasing soil biodiversity, which lead to the sustainable improvement of the agricultural system and a simultaneous increase in its resilience. The world is facing the threat of climate change and the manufacture of nitrogen fertilizers is an energy intensive process which releases greenhouse gasses into the atmosphere; in this regard, pulses can contribute to make a more rational use of nitrogen fertilizers while mitigating climate change. Furthermore, the broad diversity of pulses can play an important role in adapting to future climate change scenarios. Pulses are a critical and affordable source of plant-based protein, vitamins and very important minerals like iron, calcium, magnesium and zinc; therefore, they contribute to the food security and nutrition of many people around the world, especially for subsistence smallholder farmers. Additionally, pulses can positively impact human health, since recent research reported that their consumption can prevent and help to manage disorders such as obesity, diabetes and coronary conditions.

Despite all the benefits provided by pulses, their per capita consumption has been steadily declining in both developing and developed countries. This trend not only reflects changes in dietary patterns and consumer preferences, but it also shows the failure of domestic production to keep pace with population growth in many countries. When comparing pulses with cereals, it becomes evident that the inability of increasing pulse production is most probably the result of low investment in research, development and extension.

As mentioned above, production of pulses should, in future, be linked to population growth for the sake of having the means to provide enough plant-based protein for a 
growing world population. This is even more relevant for a large part of the world population which, for economic, health or cultural reasons, covers their protein requirements from plant products. In order to increase pulse production, research and development on pulses should come more to the forefront across the world. Researchers need to solve problems of low yields and susceptibility to some abiotic and biotic stresses. In order to achieve this, new high yielding varieties, resistant to major agricultural pests and diseases and adapted to changing environmental conditions, need to be developed. The required traits for developing new varieties adapted to different scenarios can be sourced from the large genetic resource collections available through the international genebanks network.

Breeding will therefore be a very useful tool for improving pulse production; however, new biotechnologies, both low-tech and high-tech, are needed in order to serve the interests of farmers and significantly accelerate the development of cultivars while saving time and funds needed for pulse breeding. Therefore, it is particularly appropriate that this special issue on "leguminous pulses", to celebrate the International Year of Pulses, is produced by a high standard journal which specialises in plant biotechnology. Papers presented in this volume cover different topics; the reviews give an excellent idea about the recent advances in breeding and biotechnology of legumes, including the future prospects of these techniques. Some scientists presented their results on how biotechnologies can help plants to acquire tolerance to biotic and abiotic stress and others on approaches to overcome the problem of regeneration recalcitrance. However, it is important to note the great significance of contributions dealing with underutilized pulses such as Bambara beans [Vigna subterranea (L.) Verdc.] and African yam bean [Sphenostylis stenocarpa (Hochst. ex A. Rich.) Harms] that are also being published in this issue, because underutilized species can be the source of traits required for future breeding programs.

Last but not least, I hope this special issue will trigger the interest of many scientists to initiate new scientific work oriented to solve research needs on economically important pulse crops, as well as on underutilized pulse species.

Dr. Teodardo Calles, Agricultural Officer. 